\title{
Sensitive Phonon Detection in a Spiderweb Optomechanical Resonator
}

\author{
Jessie Rosenberg, Qiang Lin, and Oskar Painter \\ Department of Applied Physics, California Institute of Technology, Pasadena, CA 91125, USA. \\ e-mail: jessier@caltech.edu
}

\begin{abstract}
We report position-squared coupling six orders of magnitude larger than previously demonstrated, allowing measurement of as few as 652 phonons and presenting a practical route toward probing of single-phonon jumps and characterization of phonon statistics.

(C) 2009 Optical Society of America

OCIS codes: (230.4685) Optical microelectromechanical devices, (230.5750) Resonators
\end{abstract}

Much recent attention in the field of cavity optomechanics [1-5] has been focused on the linear coupling between optical and mechanical modes, leading to dynamic back-action feedback [1-5] or static mechanical tuning [3]. In particular, great progress has been made towards the goal of using optomechanical effects to cool a mesoscopic-scale structure into its quantum ground state [1]. However, as the mechanical motion approaches the quantum regime, a problem becomes apparent: displacement measurements, such as are made by optical modes having linear coupling to the device mechanics, cannot measure the mechanical energy eigenstate of the oscillator [6]. An alternative has been proposed [2], utilizing a system with second-order coupling: coupling which is sensitive only to the square of the mechanical displacement, allowing for direct measurement of the system's mechanical energy eigenstate without perturbing that eigenstate due to either dynamic or quantum backaction. Here, we show a "spiderweb" double-ring resonator (shown in Fig. 1(a,b), [3]) supporting a dispersive coupling rate approximately six orders of magnitude larger than previously demonstrated [2]. With this very high coupling rate, we are able to experimentally measure phonon occupation, which has not previously been shown in a mesoscopic mechanical system.
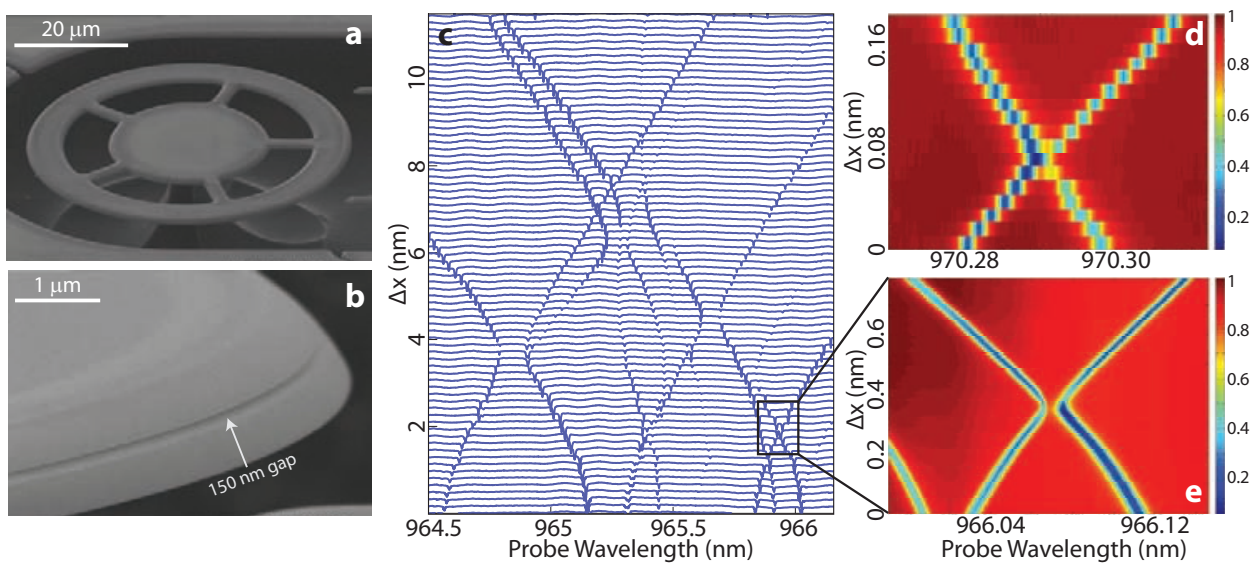

Fig. 1. (a) Scanning electron microscope image of a $54 \mu \mathrm{m}$ spiderweb resonator. (b) Scanning electron microscope image showing the gap between the rings. (c) Scanning transmission traces of the $980 \mathrm{~nm}$ band probe laser as the $1550 \mathrm{~nm}$ band pump dropped power is increased, decreasing the gap between the spiderweb rings. (d,e) Zoom-in on transmission scans of bonding/anti-bonding mode crossings showing a mode splitting $\beta$ of (d) less than $1 \mathrm{pm}$, and (e) $10 \mathrm{pm}$.

The spiderweb resonator is composed of two stacked $400 \mathrm{~nm}$-thick silica microrings, $60 \mu \mathrm{m}$ in diameter, separated by a $150 \mathrm{~nm}$ amorphous silicon spacer and supported radially by thin spokes for increased mechanical flexibility. The two coupled microdisks form a supercavity, splitting the resonances into bonding modes, even modes of the supercavity that pull the disks together, and anti-bonding modes, odd supercavity modes that push the disks apart. In the $1550 \mathrm{~nm}$ band, we design the disks such that only the bonding modes are supported, allowing for large uninterrupted tuning of the between-ring gap via the optical gradient force [3]. However, at shorter wavelengths, both bonding and anti-bonding modes are supported. When we tune a bonding and anti-bonding mode through an avoided crossing by optically modifying the ring gap (Fig. 1(d,e)), both modes experience only position-squared coupling at the crossing point; the magnitude of the position-squared coupling is given by $g_{\mathrm{OM}}^{\prime}=g_{\mathrm{om}}^{2} / \beta$, where $g_{\mathrm{OM}}$ is the linear optomechanical coupling coefficient and $\beta$ is half the frequency splitting between the two optical modes. By designing the disks such that the sidewalls are extremely vertical, we can make this splitting extremely small (Fig. 1(d)), optimizing the 


\section{JMC1.pdf}

structure for very large position-squared coupling and picometer-scale mechanical trapping [4].

Using a pump-probe scheme, we map the optical modes in the $980 \mathrm{~nm}$ wavelength band by using a high-power pump laser in the $1550 \mathrm{~nm}$ band, tuned to a bonding mode, to adjust the ring gap. This results in the dense map of mode crossings shown in Fig. 1(c). It is clear that a wide variety of mode crossings are accessible by using the pump mode to precisely set the ring gap, with mode splittings ranging from less than $1 \mathrm{pm}$ (Fig. 1(d)) to over $100 \mathrm{pm}$. With the large spiderweb resonator $g_{\mathrm{OM}}$ of $60 \mathrm{GHz} / \mathrm{nm}$ in the $980 \mathrm{~nm}$ band, this geometry shows promise for positionsquared coupling rates of up to $20 \mathrm{THz} / \mathrm{nm}^{2}$. The well-separated mode crossing characterized in Fig. 1(e) gives $g_{\text {om }}^{\prime}=$ $2.2 \mathrm{THz} / \mathrm{nm}^{2}$, six orders of magnitude larger than previously shown [2].

Modulating the intensity of the linear-coupled pump beam, which actuates mechanical motion of the rings, we can quantify the suppression of the linear optomechanical coupling at the anticrossing point by comparing the transduction at and away from the crossing point (Fig. 2(a)). The mechanical resonance frequency shown here is $7.6 \mathrm{MHz}$, increased from the intrinsic value of $700 \mathrm{kHz}$ by the optical spring effect induced by the pump laser. Normalizing to the large transduction magnitude observed in the linear regime far from the anticrossing point, the first-order transduction at the anticrossing point is suppressed by $38 \mathrm{~dB}$, limited primarily by the frequency stability of the pump laser.
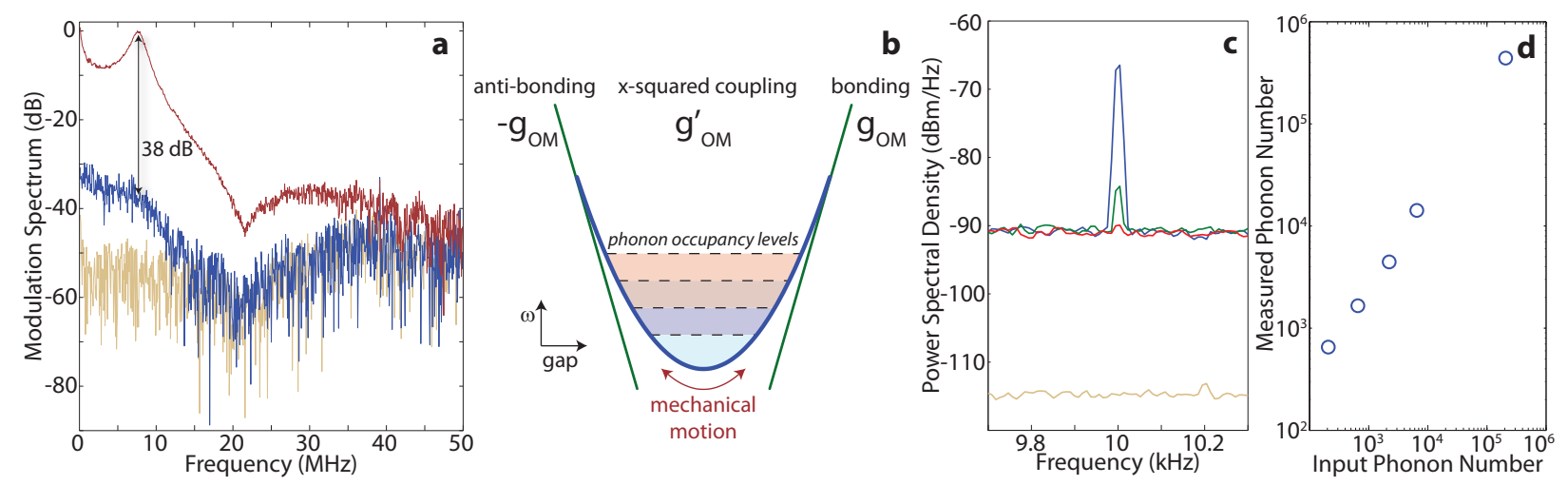

Fig. 2. (a) Pump-probe modulation of the shorter-wavelength anticrossing probe mode in Fig. 1(e), with the probe laser detuned to the red side of the resonance, at (blue) and far away from (red) the anticrossing point, normalized to the peak response. (b) Schematic of the effect of phonon occupancy on a second-order-coupled optical mode. (c) Response of the anticrossing probe mode to a phonon excitation of $2.07 \times 10^{5}$ (blue), $2.07 \times 10^{4}$ (green), and $6.51 \times 10^{3}$ (red) phonons induced by pump modulation at the mechanical resonance frequency of $7.6 \mathrm{MHz}$, amplitude-modulated at $10 \mathrm{kHz}$. (d) Linear correspondence of measured phonon number to input phonon number, measured by lock-in detection. Orange curves show the measured noise floors in $(\mathrm{a}, \mathrm{c})$.

The thermal phonon occupancy of the mechanical oscillator appears as a DC shift in the resonance of the secondorder-coupled optical mode, as illustrated in Fig. 2(b). To demonstrate the sensitivity of this measurement technique, we apply a small pump modulation at the mechanical frequency carrying an amplitude modulation at lower frequency, to dither the phonon number independently of the large thermal occupation (Fig. 2(c)). This sensitivity is limited primarily by the mechanical noise background induced by squeeze-film damping [7], significantly above the detector noise floor (orange curve, Fig. 2(c)). Performing lock-in detection we can reduce the effects of this noise background, demonstrating sensitivity down to 652 phonons (Fig. 2(d)). We attribute the discrepancy between measured and input phonon numbers to uncertainty in the optical quality factor measurements. By moving to a vacuum environment, the squeeze-film effect can be eliminated entirely, making possible single-phonon sensitivity for system parameters already within reach, in a device geometry that has shown significant promise for optomechanical cooling [5].

1. T. J. Kippenberg and K. J. Vahala, “Cavity Optomechanics: Back-Action at the Mesoscale," Science 321, 1172 (2008).

2. J. D. Thompson, B. M. Zwickl, A. M. Jayich, F. Marquardt, S. M. Girvin, and J. G. E. Harris, "Strong dispersive coupling of a high-finesse cavity to a micromechanical membrane," Nature 452, 72 (2008).

3. J. Rosenberg, Q. Lin, and O. Painter, "Static and dynamic wavelength routing via the gradient optical force," Nature Photonics 3, 478 (2009).

4. P. T. Rakich, M. A. Popovic, M. Soljacic, and E. P. Ippen, "Trapping, corralling and spectral bonding of optical resonances through optically induced potentials," Nature Photonics 1, 658 (2007).

5. Q. Lin, J. Rosenberg, X. Jiang, K. J. Vahala, and O. Painter, "Mechanical Oscillation and Cooling Actuated by the Optical Gradient Force," Physical Review Letters 103, 103601 (2009).

6. V. B. Braginsky, Y. I. Vorontsov, and K. S. Thorne, "Quantum Nondemolition Measurements," Science 209, 547 (1980).

7. M. Bao and H. Yang, "Squeeze film air damping in MEMS," Sens. Actuators, A 136, 3 (2007). 